\title{
El espíritu emprendedor. Un acercamiento al diseño independiente de moda y las oportunidades de crecimiento comercial en el contexto actual argentino
}

\section{Bertuzzi, Florencia; Escobar, Daniela [ver currículum del autor, docente de la Facultad de Diseño y Comunicación]}

\section{Resumen:}

Las oportunidades de crecimiento profesional no se encuentran solamente en el trabajo en relación de dependencia en empresas ya consolidadas en el mercado argentino, sino que el contexto socio-económico actual del país brinda la posibilidad a los nuevos profesionales del diseño de indumentaria de erigirse como emprendedores y comunicadores de sus propios productos. También ha cambiado la sensibilidad de los usuarios, quienes cada vez se muestran más interesados en consumir a conciencia, buscando productos que los diferencien en tiempos de masividad industrial, valorando las viejas técnicas de revalorización textil, como los bordados artesanales que se complementan con las nuevas tecnologías. Así, la conjunción del diseño independiente argentino con los nuevos profesionales exhibe un escenario prolífico y beneficioso, aunque también arduo para el desarrollo de nuevos emprendedores comprometidos con el progreso comercial nacional.

Palabras clave:

Emprendimiento - diseño independiente - revalorización textil - nuevas tecnologías - nuevo usuario - desarrollo comercial.

Introducción

El diseño de indumentaria, representa un engranaje muy importante en la economía de los países. La constante innovación que requieren los productos al pasar las colecciones, no solo dota a los productos de un ciclo de vida 
corto, sino que también requiere de diseñadores con capacidades y aptitudes tanto disciplinares, como interdisciplinares, para poder competir en el mercado actual de la indumentaria. La relación que existe entre los nuevos profesionales de la moda con la inserción en el mercado comercial es una temática que requiere de un análisis profundo y reflexivo para comprender de qué se trata y cómo los diseñadores emergentes se desenvuelven en el entorno al que pertenecen. El contexto actual argentino resulta favorecedor para los nuevos diseñadores de indumentaria, teniendo en cuenta que con su capacidad creativa y formación académica son capaces de llevar a cabo emprendimientos de manera independiente. Actualmente es posible crecer profesionalmente sin necesidad de trabajar en relación de dependencia en alguna empresa o marca consolidada, sino que con dedicación, ideas creativas y conocimiento se puede competir en el mercado con productos que fusionan lo artesanal con lo industrial. En primer lugar, resulta preciso determinar la diferencia entre emprendimiento y empresa. "Un emprendimiento puede ser cualquier accionar que nos planteemos para comenzar con algo nuevo, medianamente organizado. [...] Una empresa, en cambio, es un emprendimiento organizado que aspira al éxito comercial". (Spina, 2006, p. 11) En coincidencia con el pensamiento del autor antes mencionado, los diseñadores son formados académicamente desde la creatividad, muchas veces sus propuestas se fundamentan desde el aspecto estético o comunicacional y no desde el comercial, por lo que no resulta casual encontrar en el mercado propuestas creativas sumamente interesantes desde lo conceptual que flaquean en la índole comercial. En el caso puntual de los emprendimientos de moda es muy usual que esto suceda debido a que su concepción está basada, como plantean Saviolo y Testa (2013), en habilidades artísticocreativas $\mathrm{y}$, por lo tanto, producen y venden productos creativos que deben ser acordes a las necesidades del mercado para que puedan ser vendidos con facilidad. En relación a este tipo de productos, como por ejemplo la indumentaria, las autoras mencionadas con anterioridad señalan:

Este producto se crea en un contexto que sintetiza arte, artesanía y gestión. La concepción y la producción de un bien creativo absorben diferentes recursos y habilidades de estos tres ámbitos: del arte, la prioridad de la visión; de la artesanía, la acción y la manualidad; de la gestión, el pensamiento estratégico. (Saviolo y Testa, 2013, p. 50)

Es en este último punto, el de la gestión y la comercialización, donde los diseñadores y emprendedores del rubro de la moda deben contemplar la posibilidad de trabajar de manera interdisciplinaria con profesionales del área del marketing y la publicidad.

El contexto actual argentino: masividad, identidad e independencia

La Argentina se ha caracterizado por ser un país que ha sufrido dificultades económicas, crisis inflacionarias y devaluaciones en repetidos períodos de la historia. En momentos en los que la economía ha ido mejor, tanto los consumidores como el mercado local, se han visto mucho más seducidos por los productos importados que por los nacionales, hasta que la gran crisis económica del 2001 reactivó la industria nacional de la mano de profesionales creativos y capacitados para llevar a cabo un cambio de paradigma en relación al consumo. Hasta aquel momento, grandes firmas internacionales lideraban el mercado local, como así también las marcas de indumentaria nacional producían con materias primas importadas teniendo en cuenta que la producción nacional estaba prácticamente paralizada. En diciembre del 2001 el dólar se disparó y la gran devaluación empeoró la crisis social y económica del país. Las empresas extranjeras de indumentaria se vieron en la obligación de retirarse del mercado local, dado que las ventas cayeron abruptamente por la suba desmedida del dólar. A partir de este contexto tan poco favorable tanto a nivel econó- mico como social, se comenzó a reactivar la industria 
nacional y comenzaron a reabrirse fábricas que se encontraban cerradas por las políticas económicas de la década del 90, las cuales favorecían ampliamente a las importaciones. Asimismo, la gran crisis económica trajo consigo despidos y el desempleo aumentó considerablemente, por lo que quienes sabían algún oficio o contaban con un pequeño capital para invertir, decidieron iniciar sus propios emprendimientos. En dicho contexto nace el diseño de autor, de la mano de diseñadores de indumentaria que con mucha creatividad y pocos recursos monetarios reactivaron el sistema de la moda argentino.

El «Diseño de indumentaria de autor» es un segmento que forma parte de la cadena de valor textil e indumentaria de la Argentina. Se destaca por su innovación y originalidad tanto en el producto final como en las diferentes instancias del proceso proyectual y productivo. Sus creaciones no siguen las tendencias impuestas por los grandes centros de moda, por el contrario, producen bienes que comunican una identidad propia que se nutre del entorno geográfico, productivo y cultural. (Marino, P; Marré, S y Mon, L, 2014, p. 13)

El contexto actual de la moda argentina se encuentra integrado, a modo de generalización, por el Diseño de Autor, el Diseño Independiente y la moda masiva. En esta última se incluyen marcas que se dedican a la fabricación de prendas $100 \%$ industrializadas y a gran escala, las cuales responden a las tendencias impuestas por los grandes centros de moda. El Diseño Independiente, en cambio, comparte características tanto del diseño de autor como de la moda masiva. De esta última comparte la idea de concepción de productos comerciales, es decir, no se trata de una propuesta meramente conceptual aunque tampoco se rige estrictamente por seguir las tendencias. Este contexto, no solamente ha favorecido la proliferación de pequeñas y medianas empresas de producción nacional, sino que también ha cambiado la sensibilidad de los usuarios, quienes cada vez se muestran más interesados en consumir a conciencia, buscando productos que los diferencien en tiempos de masividad industrial. Por lo tanto, los nuevos diseñadores ya no se ven obligados a trabajar únicamente en relación de dependencia en empresas consolidadas en el mercado local, sino que tienen la posibilidad de erigirse como emprendedores siendo ellos mismos los productores y comunicadores de sus creaciones.

Los emprendimientos comparten la particularidad de ser una forma de autoempleo, a la vez que son auto gestionadas por los propios diseñadores, quienes llevan adelante el desarrollo creativo y los lineamientos empresariales para alcanzar la sostenibilidad de las empresas. (Marino, et al., 2014, p. 13)

Estas formas alternativas de crecer profesionalmente en el ámbito del diseño han cambiado también las modalidades de comercializar los productos para adaptarse a las necesidades económicas, tanto de los usuarios como de los emprendedores. La presencia en Internet resulta ser casi obligatoria tanto para las marcas consolidadas, como para las que recién se inician en el mercado, siendo para muchas de estas últimas el único espacio en el que cuentan con el contacto con el usuario. Según Marino et. al. (2014), en su diagnóstico de diseño de autor independiente realizado por el Instituto de Tecnología Textil junto con la Fundación ProTejer, se observa en el territorio argentino que desde 2010 hasta 2014 las empresas de diseño han demostrado una permanente adaptación de sus estrategias comerciales y comunicacionales. Se demuestra que las pequeñas empresas buscan optimizar y ampliar su público, mediante el intenso uso de las redes sociales, convirtiéndose éstas últimas en herramientas eficaces. El surgimiento de redes sociales activas como Facebook, Instagram, Periscope, Twitter, entre otras, permiten que las marcas emergentes compartan a cada momento no sólo las tomas fotográficas de una colección, sino el paso a paso del desarrollo del producto, desde la elección de un concepto, video o tema musical como punto de inspiración, pasando por la publicación de fotografías que expongan la intervención o selección de los textiles hasta la realización y tomas de un desfile. De esta manera, 
se involucra a los usuarios en el proceso creativo de la marca generando una fidelización con los consumidores y un constante feedback con los clientes, pudiendo conocer sus comentarios y opiniones sobre lo que la marca ocasiona. Asimismo, la aparición de tiendas virtuales, a las cuales se puede acceder con una mínima inversión, permite a las nuevas marcas de moda independiente comercializar sus productos de forma inmediata, expandiendo sus horizontes hacia nuevos mercados, ya que otorgan la posibilidad de adquirir productos desde cualquier lugar del país o del mundo, para luego ser enviados por correo y que el cliente los reciba cómodamente en su domicilio. Es posible determinar que cada vez más marcas independientes deciden no contar con un local a la calle, sino que prefieren dejar sus productos en consignación en locales afines a la marca o multimarcas, como también organizar showrooms, participar de ferias de dise- ño o vender por Internet. Esta situación es el resultado de los ajustados presupuestos que manejan los emprendedores: mantener un local al público requiere de mucha inversión y grandes cantidades de ventas para poder cubrir los gastos fijos. Por este motivo, muchas marcas de diseño independiente terminan siendo itinerantes, ya que no cuentan con un lugar fijo en el cual ubicar sus productos: van de feria en feria, tratando de llegar a nuevos usuarios cada fin de semana. Asimismo, los emprendedores cuentan también con la posibilidad de presentar sus proyectos a incubadoras de empresas, de las cuales reciben las herramientas necesarias para que sus proyectos se desarrollen, crezcan y, finalmente, se consoliden como empresas autosustentables.

Tal y como lo plantea Spina (2006), en muchos países el desarrollo de incubadoras de empresas ha sido importantísimo para la reactivación de la economía, ya que sin su ayuda, dichas firmas no podrían haberse sostenido en el tiempo como tales. La Argentina, a partir del año 2002 cuenta con su incubadora de proyectos: Incuba que se sostiene gracias al apoyo de la Secretaría de Cultura del Gobierno de Buenos Aires y al Centro Metropolitano de Diseño. Incuba cuenta con un programa de convocatorias periódicas a través de las cuales los emprendedores pueden presentar sus propuestas.

Según sea la organización de la incubadora, serán los requisitos para acceder a la incubación y la forma en que se pacte el egreso de la misma. Algunas incubadoras exigen que la empresa devuelva los servicios recibidos, con el aporte económico de un porcentaje de sus ingresos. Otras incubadoras no exigen aportes económicos, solamente piden el compromiso del emprendedor para con nuevos emprendedores que participen de la incubación. (Spina, 2006, p. 60)

La posibilidad de que un proyecto quede seleccionado en la incubadora resulta muy beneficioso para el emprendimiento, teniendo en cuenta que de esa manera el camino del emprendedor toma un rumbo más positivo debido a que los errores u obstáculos que suelen presentarse son menores en comparación con los que se presentan en el camino de quienes emprenden de manera independiente y solitaria, teniendo que recurrir a profesionales ajenos al diseño de indumentaria para subsanarlos. Está claro que quienes deciden emprender en el rubro de la moda de manera independiente, deben tener objetivos claros, como también investigar y analizar el mercado en función de poder ofrecer productos acordes a las necesidades de los usuarios. Asimismo, el emprendedor debe estar capacitado para poder dar soluciones creativas a los problemas que se pueden presentar a medida que el emprendimiento va creciendo. Spina (2006) afirma que los diseñadores tienen un concepto de creatividad basado en la morfología y en la estética de los productos o servicios que pueden ofrecer y al respecto sugiere:

Debemos ejercitarnos en la creatividad aplicada a la vida diaria de nuestro emprendimiento, en todas las facetas que éste comprende. En buscar soluciones creativas a problemáticas comerciales, de producción, de 
posicionamiento, de alianzas estratégicas, de financiamiento, de asociatividad. En definitiva, nos diferenciaremos de nuestros competidores, justamente, con los procesos creativos aplicados en donde los necesita nuestro emprendimiento, transformando debilidades en fortalezas y aprovechando nuestras propias fortalezas para potenciarnos aún más. (Spina, 2006, p. 14)

El hecho de que cada vez más sean los diseñadores que deciden iniciarse en el campo de la moda a través de un proyecto personal ha impulsado la industria nacional, así como también la revalorización de oficios y técnicas que habían quedado en el pasado.

Nuevo pensamiento: adaptación del diseñador a las nuevas exigencias de los usuarios.

Resulta sustancial destacar que el usuario argentino hasta la crisis del 2001 tenía una especial preferencia por los productos importados. Podía adquirirlos ya que su economía de bolsillo se lo permitía y la calidad de los mismos era superior a los productos nacionales. Existe una toma de conciencia a nivel global por parte de los usuarios en relación al consumo. Posiblemente esta nueva forma de pensamiento tenga que ver con la crisis económica mundial y haya sido el resultado de la adaptación que tuvieron que hacer los nuevos dise- ñadores para poder seguir adelante con sus proyectos de moda. El consumo ya no se rige por el simple hecho de consumir y acumular, sino que ha cambiado de acuerdo a las nuevas exigencias del mercado y principalmente de los usuarios, quienes tienen mayor conocimiento de lo que se usa, de materialidades, de nuevas tecnologías, como también muestran un aspecto más nostálgico hacia viejas técnicas u oficios. La labor artesanal va ganando terreno en el mercado y los usuarios la valoran cada vez más por sobre los productos $100 \%$ industrializados, lo que favorece a los diseñadores independientes que deciden iniciar un emprendimiento con poco capital. Los nuevos emprendedores del diseño de modas optan cada vez más por la investigación y experimentación con materialidades textiles inteligentes utilizadas en principio para otros fines como las telecomunicaciones, deportes o medicina, para luego adaptarlas a la indumentaria casual utilizada para actividades diarias. Eligen experimentar con materiales nanotecnológicos para crear prendas que cumplan con funciones específicas o que aporten confort a la vida diaria, como por ejemplo, prendas autolimpiables, protección contra alergias, con propiedades cosméticas o que permitan la carga de celulares y artículos electrónicos. También, la inventiva de los diseñadores emergentes se expande cuando se vincula con la cuestión ambiental. La utilización de fibras naturales orgánicas certificadas, como el algodón y bambú ya es una práctica muy difundida y no es suficiente, pues ahora también se busca revalorizar y utilizar desechos textiles para crear nuevas prendas, como descoser y rediseñar prendas descartadas y en desuso. De esta manera, además de comprometerse de forma ecológica y sustentable con el entorno, se generan diseños inéditos, colecciones limitadas, las cuales permiten a los consumidores de moda optar por indumentos únicos e irrepetibles. En el mismo sentido, se ha puesto en evidencia -especialmente en América Latina- una inclinación por parte del diseñador hacia diversas culturas ancestrales pertenecientes a su territorio. De esta inquietud se produce la investigación de métodos y técnicas de tejido ancestrales de la producción artesanal manual, en contraposición a los métodos de producción industriales seriados. Así pues, se posee una marcada intención por revalorizar técnicas ancestrales y dignificar el trabajo de los artesanos, poniendo en valor la producción de artículos de indumentaria artesanales, únicos y representativos de cada país. La preocupación por el medio ambiente y la conciencia general de los consumidores sobre el uso responsable de los recursos que el planeta otorga, de la mano de prácticas como el reciclaje y la sustentabilidad, han llegado al mundo de la moda. Éstas prácticas no sólo resultan diferenciadoras para las marcas emergentes, sino que también corresponden a nuevas exigencias de un usuario que necesita conocer lo que está usando y elige comprar productos que cumplan con requisitos 
amigables con el medio ambiente. Dentro de este contexto, los diseñadores emergentes se encuentran frente a nuevos desafíos que incluyen no sólo producir diseño indumentario en base a la creatividad, sino tener en cuenta las nuevas necesidades, exigencias, carencias de los consumidores de moda. Por lo tanto, el reto reside en comprender, analizar el contexto y a los mismos usuarios, para poder adentrarse en el mercado de forma efectiva y diferenciadora. En la actualidad, se observan más emprendimientos de moda que se comprometen con el cuidado medioambiental, la conservación y revalorización de antiguas técnicas de producción, el trabajo de sastrería artesanal, los bordados manuales y la intervención o customización de prendas. Cada día el diseñador parece tener la necesidad, acompañada de su capacidad creativa, de ser parte del proceso productivo de las prendas, además de ser quien ser encarga de la conceptualización de la propuesta y de generarla morfológica y estéticamente. El poder combinar las formas industriales de confección (las cuales aceleran los tiempos de producción y mantiene al emprendimiento en constante movimiento y crecimiento) con detalles artesanales o manuales, resulta una combinación favorecedora, ya que dota al producto de una identidad propia y diferenciadora en relación a las prendas que se puedan encontrar en el mercado. Detrás de cada prenda con dichas características existe una historia, una persona o diseñador que le dedicó horas de trabajo a cada prenda para que finalmente llegue a las manos del usuario. El poder ofrecer un producto de diseño y calidad, acorde a las necesidades del consumidor y en concordancia con la ideología del diseñador, es solamente una parte importante del emprendimiento, se requiere, principalmente, poder comercializarlo de manera óptima de modo que la pequeña empresa pueda crecer favorablemente con el tiempo, adaptándose a los cambios contextuales y/o económicos del país.

Resurgimiento de la periferia: el diseño invade todo el país

Además de analizar la importancia del proceso productivo y el engranaje emprendedor de la mano de las causas y consecuencias del surgimiento del diseño de autor e independiente argentino, es un punto clave definir la localización de los emprendimientos en las diferentes provincias y ciudades que comprenden el territorio argentino. Actualmente la actividad de diseño indumentario independiente denota una expansión notable hacia puntos periféricos de la Argentina, siendo que en los inicios de la actividad, se concentraban la mayoría de pequeñas empresas en la Ciudad Autónoma de Buenos Aires y el conurbano bonaerense.

Lejos de su inicio académico porteño y de su difusión mediática, la indumentaria de autor no sólo se hace en Buenos Aires, sino que hoy está distribuida en 20 provincias. La federalización del diseño es un hecho que se ve en el alto porcentaje de empresas que funcionan en el interior: $72 \%$, mientras que la ciudad de Buenos Aires tiene un 28 por ciento. Pero ese porcentaje concentra la tercera parte de las empresas de diseño de la Argentina y factura un $88 \%$ del total de ingresos que obtienen en este segmento en todo el país. (Piña, 2015)

En este punto, se observa el importante crecimiento de la actividad en zonas alejadas de la capital, convirtiéndose en una actividad económica impulsora de nuevos profesionales radicados en diferentes puntos del país, valorizando el proceso creativo y de diseño perteneciente a distintas provincias, las cuales aportan nuevas visiones y colecciones de moda inéditas. Siguiendo el artículo publicado por Piña (2015) para el diario La Nación, entre las 77 empresas de diseño en la Capital se destacan Cora Groppo, Garza Lobos, Juana de Arco, Kostüme, Dappiano, Min Agostini, Pesqueira, Tramando y Seco, siendo éstas las marcas más conocidas, pero asimismo, no sólo se encuentran estos emprendimientos destacados porteños, sino que se erigen con cada vez más fuerza otros diseñadores que residen y exponen sus creaciones en ciudades como Rosario, Santa Fe, Córdoba, La Pampa, San Miguel de Tucumán, Salta, Jujuy, Santiago del Estero, Catamarca, Mendoza, San 
Juan, San Luis, Neuquén, Río Negro y Chubut. Principalmente se destacan diseñadores como: Manuela Rasjido, Lucía Belaustegui, Uchi Bolcich, Santos Liendro, Quier, Bossini Pithod, entre otros. En el caso de la marca rosarina Quier, por ejemplo, se vincula con la moda lenta, el slow fashion y criterios sostenibles para producir sus prendas. En concomitancia, Manuela Rasjido, oriunda de los Valles Calchaquíes en la provincia de Catamarca, expone prendas relacionadas con el concepto de vida slow, que van de la mano con el trabajo artesanal, siendo importante la producción de teñido a mano con elementos naturales vegetales, sin agregados químicos. Lucía Belaustegui, nacida en la provincia de Corrientes, busca rescatar la herencia de su territorio en sus colecciones, teniendo como fuente de inspiración la naturaleza y las formas orgánicas que la rodean para la posterior creación de prendas personalizadas. La diseñadora Uchi Bolcich, busca también inspiración en su ciudad natal, Bariloche, y utiliza una conjunción del diseño de indumentos a mano alzada con el uso de la tecnología láser para crear puntillas, tramas y moldería para plasmar sus diseños. Bajo la misma línea, la marca Santos Liendro plasma la identidad del noroeste argentino, reflejando la belleza de la naturaleza circundante en el diseño de indumentaria, zapatos, accesorios y una línea para el hogar presente en la ciudad de Salta. La marca de diseño de indumentaria masculina oriunda de Mendoza, Bossini Pithod, mediante la utilización del patchwork como técnica fundamental en sus colecciones, toma además como disparador, al hombre argentino y su indumentaria característica para rediseñar indumentaria urbana en base al vestido autóctono de distintas regiones del país y de este modo revalorizarlo. Además de favorecer la economía de cada provincia en materia de ingresos, los nuevos diseñadores emergentes que se destacan en todo el país, no sólo diseñan de forma creativa y funcional, sino que buscan la diferenciación mediante la adopción de técnicas de tejido ancestral, producción artesanal y la búsqueda de materiales propios de cada territorio. Asimismo, se denota una búsqueda de dar valor a lo propio, tomarlo como punto de inspiración y también dar importancia a la labor artesanal de cada región, promulgando valores de sostenibilidad y comercio justo. De este modo, se genera una revalorización de todas las regiones del territorio argentino, siendo el diseño independiente, un motor impulsor de la creatividad argentina hacia el mundo.

\section{Nuevos horizontes: emprender creativamente}

El ingreso al mercado pone a prueba los sueños de los emprendedores. El comienzo de la empresa plantea el desafío de legitimar la viabilidad del proyecto empresarial demostrando su capacidad para comercializar el producto/servicio, enfrentar a la competencia, superar los problemas asociados a la gestión de recursos humanos, manejar adecuadamente las limitadas fuentes de financiamiento disponibles y, en la medida que se consiga sobrevivir y expandirse. (Piazza, 2006, p. 23)

Desde esta perspectiva, el diseñador que se inserta en el mercado luego de haber estudiado una carrera universitaria, no posee las aptitudes necesarias para erigir una emprendimiento redituable y próspero que se mantenga a lo largo del tiempo. Sin embargo, los nuevos profesionales son conscientes de que poseen la capacidad creativa pero que la visión comercial y comunicacional de un proyecto independiente les resulta difícil de abarcar. De igual modo, conocen y valoran la importancia de las perspectivas antes mencionadas para lograr que su pequeña empresa prospere. De esta manera, surge la necesidad de integrar equipos dinámicos de trabajo, siendo clave la integración de otros profesionales a la estructura empresarial, ya sea como empleados eventuales o estables. Cuando el nuevo emprendedor se encuentra en la fase inicial del proyecto, es habitual que se encuentre con los recursos monetarios acotados para algunas de las etapas que componen el proceso de generar un emprendimiento. Generalmente, gran parte del presupuesto lo invierten en la fase productiva, en la creación y confección de las prendas, quedando un presupuesto demasiado acotado para invertir en la 
comercialización y comunicación. Puede resultar favorecedor recurrir a lo que se conoce como alianzas estratégicas o trabajos interdisciplinarios, a partir de los cuales las partes intervinientes en dicha alianza obtienen algún tipo de beneficio, ya sea de índole económica o de otro tipo.

Se puede considerar como un aliado estratégico, a una persona, institución, organismo público o empresa, con la cual podamos generar un vínculo que sirva a ambas partes para desarrollar alguna actividad, con el concepto de «ganar-ganar», o sea que ambos salgan favorecidos por esa alianza. (Spina, 2006, p. 58)

Por lo tanto, es posible determinar que el diseñador no trabaja solo, no se construye como una única persona que abarca todas las tareas, sino que propone un plan de trabajo de la mano con otras personas que complementan su trabajo. Es en este caso, donde surge el concepto de trabajo multidisciplinario, mediante el cual el diseñador de indumentaria puede completar su labor creadora con la visión comercial del marketing, la publicidad y la comunicación eficaz de sus diseños. La inclusión de profesionales de otras ramas creativas, como el diseño gráfico, packaging y fotografía agregan valor a las producciones indumentarias. Bajo la misma perspectiva, teniendo como objetivo generar ganancias y reconocimiento de las marcas de indumentaria en el imaginario público, surge la posibilidad comercial de realizar alianzas estratégicas. Por definición una alianza estratégica "[...] es una estrategia popular que ocurre cuando dos o más empresas integran una asociación o consorcio temporal con el propósito de aprovechar alguna oportunidad" (David, 2003, p. 177). En este caso surgen numerosos ejemplos de alianzas estratégicas en materia de moda e indumentaria presentes en el territorio argentino, mediante las cuales las grandes, medianas y pequeñas empresas de diseño buscan beneficiarse a través de la asociación entre empresas para promover el consumo de sus mercancías, como así también generar productos novedosos y darse a conocer a públicos ajenos a los que normalmente consumen los productos de cada marca aliada. "El fenómeno no es nuevo: hace varios años que, consagrados diseñadores internacionales, realizan colecciones cápsula para cadenas de ropa populares o de consumo masivo". (Carlos, 2015) La marca de indumentaria masculina Félix, se alió con la empresa de telefonía y electrónica Samsung en el año 2011 para comercializar bike bags, pequeños bolsos confeccionados en lona con tiras de cuero que permiten transportar todos los efectos de tecnología personal en la bicicleta. La empresa Samsung en el mismo año también se alió con Lupe, una empresa de indumentaria y accesorios femenina para crear una pequeña colección limitada de mini bags confeccionadas en cuero para guardar celulares. En este caso, una gran empresa reconocida a nivel mundial, Samsung, realiza una alianza estratégica con empresas de diseño argentinas que producen indumentaria de hombre y mujer, Lupe y Félix, para crear un producto nuevo y de edición limitada para generar nuevos clientes de la mano con la aplicación del diseño y las tendencias presentes a los accesorios relacionados a la tecnología. Otro caso destacado es la propuesta Diseño para todos, impulsada por la cadena de supermercados Jumbo, mediante la cual se pretende acercar el diseño de autor al público masivo a través de la venta de indumentaria industrial en ediciones limitadas a bajo costo, intervenida por diseñadores argentinos. El programa ha sido implementado desde el año 2005, y ha contado con la participación de diseñadores de autor e independientes como Jessica Trosman, Benito Fernández, Seco, Las Oreiro, Picnic y Pesqueira. Del mismo modo, la marca argentina de indumentaria adolescente Muaa se alió con los reconocidos diseñadores de autor Pablo Ramirez y Mariano Toledo para comercializar pequeñas colecciones limitadas cápsula que integren las creaciones con gran impronta de diseño que proponen los diseñadores al público teen. Sin embargo, las alianzas estratégicas no se dan únicamente entre grandes marcas reconocidas en conjunto con diseñadores de autor o independientes. Otra forma de asociación se da entre marcas de indumentaria y celebridades o trendsetters que estén en boga en el momento. Tal es el caso de las 
denominadas it girls, quienes imponen un estilo de moda: Candelaria Tinelli con la marca Muaa, Lucía Celasco con la firma adolescente Como quieres que te quiera, Calu Rivero con Complot y Florencia Torrente con la marca de accesorios y marroquinería Lázaro. En este caso, las marcas se benefician transmitiendo a través de la creación de una pequeña colección cápsula o de edición limitada, el carácter y personalidad de cada una de las celebridades, las cuales pueden poseer o no un estilo similar al de la firma representada. De tal modo, la marca de indumentaria demuestra un acercamiento a las tendencias latentes y se acerca a su público. La indumentaria masculina no queda fuera de este tipo de alianzas. La marca Garçon García convocó al músico Dante Spinetta para dar origen a la colección cápsula Volans, la cual está integrada por 25 piezas de diseño confeccionada con materiales de primera calidad. En la Universidad de Palermo también se han generado numerosos casos de alianzas estratégicas, mediante las cuales se ha impulsado a los alumnos a demostrar sus creaciones en un nivel profesional a través de concursos. Tal es el caso de la asociación con la empresa Ritex, que produce tejidos de punto o la marca Nube, productora de lanas, quienes donaron materiales para que los estudiantes puedan crear diseños innovadores y mostrarlos en la Semana de la Moda de la Universidad de Palermo. Las marcas Stone y la diseñadora Ágatha Ruiz de la Prada, también han participado del intercambio, solicitando a los estudiantes de diseño la realización de prendas inspiradas en el estilo de la marca para poder comercializarlas en sus locales. De esta manera, las firmas se relacionan con los futuros profesionales, otorgando una visión fresca y renovada a marcas que lideran hace mucho tiempo el mercado del diseño de autor español y la producción de prendas de denim en la Argentina. Teniendo en cuenta la investigación de los usuarios y sus necesidades, las nuevas ventajas de mercado ofrecidas por los países latinoamericanos, las nuevas opciones de desarrollo textil, la posibilidad de utilizar equipos multidisciplinarios y la oportunidad de utilizar todo tipo de alianzas estratégicas, conjugan el nuevo horizonte de los diseñadores emergentes, los cuales poseen numerosas herramientas eficaces y oportunas para poder concretar un negocio redituable, exitoso y que además comunique la esencia propia creativa de cada uno de ellos.

\section{Conclusión}

“[...] lo interesante de un emprendimiento no es 'llegar', sino 'recorrer el camino'. Y ese camino se recorre a diario, se vive cada día, y por lo tanto se debe disfrutar en cada momento". (Spina, 2006, p. 62) A modo de cierre se puede expresar que el camino para lograr emprender un negocio de moda en la República Argentina no es fácil y confortable, sino todo lo contrario, resulta ajetreado, arduo y complejo. Pero, sin embargo, en el contexto actual resulta importante y clave destacar que no sólo es posible, sino que las condiciones están dadas para que los nuevos profesionales del diseño de autor e independientes puedan emprender negocios exitosos y destacarse no sólo en el país sino también a nivel mundial. La voluntad y energía puesta en el trabajo en pos de resultar reconocido, destacado por su estilo propio y exitoso en materia económica, puede describirse como la esencia y el espíritu de los nuevos emprendedores del diseño de modas.

Los grandes emprendedores sueñan, pero sobre todo hacen, hacen y hacen. Muchos de ellos tienen grandes capacidades intelectuales, pero su foco siempre está puesto en lograr resultados concretos. [...] Todos tienen una clara visión y esperanza hacia dónde pueden y quieren llevar su futuro y el de su empresa. (Freyre, 2005)

Al decir que las condiciones están dadas, se habla no sólo de factores económicos y de proliferación de creativos competentes en todas las áreas de diseño, no obstante se considera que la identidad propia del diseño argentino es una ventana hacia el exterior, es una visión creativa diferente, única que expone las riquezas de un país en el cual hay mucho que expresar y contar. La creatividad no reside solamente en la capital del país, sino 
que se encuentran numerosos diseñadores y creativos en todo el territorio, quienes exponen productos, ideas y visiones inéditas en conjunto con una identidad marcada y arraigada con lo que su tierra inspira. La Argentina al ser un territorio tan amplio, vasto de recursos y paisajes diferentes, alberga no sólo diferentes ecosistemas sino también diferentes culturas, que aportan en materia de moda y diseño, creativos competentes y destacados que representan a cada una de esas culturas de manera fidedigna. Los constantes y repetitivos golpes y crisis en materia económica y política en las cuales se ve inmerso el pueblo argentino ha generado una resiliencia y adaptación magnífica a los cambios, siendo éste un motor impulsor que fomenta y exterioriza las capacidades profesionales y creativas de los diseñadores emergentes (como así también en otras profesiones y oficios) para generarse su propio empleo y poder erigirse como profesionales independientes. Al no poder acceder fácilmente a empleos o puestos de trabajo en relación de dependencia, ya sea por motivos económicos, de mucha competencia laboral o la carencia de empresas que contraten profesionales, se genera una proliferación de pequeñas y medianas empresas independientes, familiares con ímpetu propio por erigirse e insertarse en el mercado local, latinoamericano e incluso mundial. Dicho de otro modo, la necesidad de emprender es más que un deseo, es una vocación que surge de la mano de oportunidades y una marcada visión de crecimiento. Es por esto, que el futuro de los diseñadores de moda argentinos independientes, reside en generar múltiples aptitudes, siendo importante no centrarse únicamente en el proceso de diseño y producción de colecciones de moda. Por consiguiente, en el contexto actual para lograr ser un emprendedor y diseñador destacado se precisa de profesionales que sepan observar y reflexionar sobre los nichos de mercado, complementar las cualidades creativas propias de cada uno de ellos en beneficio de los usuarios o consumidores, comprender los beneficios comerciales de las alianzas estratégicas y el trabajo multidisciplinario, y sobre todo, proponer una mediante el diseño, una identidad marcada y diferenciadora para poder convertirse en los nuevos dueños y representantes del mercado de la moda argentino.

\section{Referencias Bibliográficas}

David, F. (2003) Conceptos de administración estratégica. $9^{\circ}$ ed. Naucalpan de Juárez: Pearson Educación de México S.A. Carlos, M. (2015) Alianzas estratégicas, diseño por y para todos. Buenos Aires: Revista FH [Revista en línea]. Recuperado el 29/02/2016 de: http://www.revistafh.com.ar/?p=1419 Freyre, A. (2005) Pasión por emprender: De la idea a la cruda realidad. Buenos Aires: Grupo Editorial Norma.

Marino, P; Marré, S. y Mon, L. (2014) Diseño de indumentaria de autor en argentina: diagnóstico productivo e impacto económico basado en la Encuesta Nacional de Diseño de Indumentaria de Autor 2014. Buenos Aires: INTI y Fundación Pro Tejer. Piazza, J. (2006). El diseño como negocio. Un análisis conciso de la problemática de emprender la profesión del diseño, vista como negocio. Buenos Aires: CommTOOLS. Piña, D. (2015) El diseño vende. Buenos Aires: Diario La Nación. Recuperado el 29/02/2016 de: http://www.lanacion.com.ar/1771341-eldiseno-vende Saviolo, S y Testa, S. (2013) La gestión de las empresas de moda. Barcelona: Gustavo Gili. Spina, M. (2006) Guía práctica para emprender en el diseño: Lo que tenemos que conocer para desarrollar nuestro emprendimiento de servicios o productos de diseño. Buenos Aires: CommTOOLS.

\section{Abstract:}

In the Argentine market, the professional growth opportunities are not exclusive to pre-existing consolidated companies. The current socio-economic context of the country offers the possibility for new fashion design professionals to establish themselves as entrepreneurs and communicators of their own products. It has also 
changed the sensitivity of users who are increasingly interested in consuming conscientiously; consumers are looking for products that differ in industrial massiveness, while simultaneously valuing the old techniques of textile revaluation as artisanal embroidery complemented by new technologies. Thus, the combination of Argentine independent design with new professionals shows a prolific and beneficial scenario, yet it problematizes the development of new entrepreneurs committed to national commercial progress.

Key words:

Entrepreneurship - independent design - textile revaluation - new technologies - new user - commercial development.

\section{Resumo:}

As oportunidades de crescimento profissional não estão somente no trabalho em relação de emprego em empresas já consolidadas no mercado argentino, senão que o contexto sócio econômico atual do país dá a possibilidade aos novos profissionais do design de indumentária de estabelecerem-se como empreendedores e comunicadores de seus próprios produtos. Também mudou a sensibilidade dos usuários, que cada vez mostram-se mais interessados em consumir com consciência, procurando produtos que os diferencem em tempos de massificação industrial, valorando as velhas técnicas de revalorização têxtil, como os bordados artesanais que se complementam com as novas tecnologias. Deste modo, a conjunção do design independente argentino com os novos profissionais exibe um cenário prolífico e favorável, embora difícil para o desenvolvimento de novos empreendedores comprometidos com o progresso comercial nacional.

\section{Palavras chave:}

empreendimento - design independente - revalorização têxtil - novas tecnologias - novo usuário desenvolvimento comercial.

El espíritu emprendedor. Un acercamiento al diseño independiente de moda y las oportunidades de crecimiento comercial en el contexto actual argentino fue publicado de la página 41 a página53 en Cuadernos del Centro de Estudios de Diseño y Comunicación № 64 